\title{
Genetic Analysis of LRRK2 R1628P in Parkinson's Disease in Asian Populations
}

\author{
Yuan Zhang, ${ }^{1,2}$ Qiying Sun, ${ }^{2,3,4}$ Minhan Yi, ${ }^{5,6}$ Xun Zhou, ${ }^{1}$ Jifeng Guo, ${ }^{1,3,4,5}$ Qian Xu, \\ Beisha Tang, ${ }^{1,3,5}$ and Xinxiang Yan ${ }^{1,3,4}$ \\ ${ }^{1}$ Department of Neurology, Xiangya Hospital, Central South University, Changsha, Hunan 410008, China \\ ${ }^{2}$ Department of Geriatrics, Xiangya Hospital, Central South University, Changsha, Hunan 410008, China \\ ${ }^{3}$ National Clinical Research Center for Geriatric Diseases, Changsha, Hunan 410078, China \\ ${ }^{4}$ Key Laboratory of Hunan Province in Neurodegenerative Disorders, Central South University, Changsha, Hunan 410008, China \\ ${ }^{5}$ State Key Laboratory of Medical Genetics, Changsha, Hunan 410078, China \\ ${ }^{6}$ Institute of Information Security and Big Data, Central South University, Changsha, Hunan 410083, China
}

Correspondence should be addressed to Xinxiang Yan; xxyan1268@126.com

Received 16 February 2017; Revised 10 June 2017; Accepted 29 August 2017; Published 25 October 2017

Academic Editor: Jan Aasly

Copyright (C) 2017 Yuan Zhang et al. This is an open access article distributed under the Creative Commons Attribution License, which permits unrestricted use, distribution, and reproduction in any medium, provided the original work is properly cited.

\begin{abstract}
Although the etiology of Parkinson's disease (PD) remains unclear, there is increasing evidence of genetic factors contributing to the onset of PD. Various mutations and risk variants of the gene LRRK2 have been reported, but the association between LRRK2 $\mathrm{R} 1628 \mathrm{P}$ and PD is still inconsistent. Thus, we conducted a meta-analysis to determine the potential relationship between R1628P and PD. Our study sample was an aggregate of 17 publications, which in total consisted of 9,275 PD patients and 8,114 controls. All of these articles are of high quality according to NOS, and there was no obvious reporting bias or heterogeneity. In a general Asian population, the pooled OR of the risk genotype contrasts was 1.83 (95\% CI: 1.57, 2.13). When stratified by ethnicity, the pooled ORs were 1.84 (95\% CI: 1.56, 2.18) in a Chinese population and 1.79 (95\% CI: 1.27, 2.52) in a non-Chinese population. Our study suggests that LRRK2 R1628P appears to be a risk factor for PD in Asian populations, both Chinese and non-Chinese.
\end{abstract}

\section{Introduction}

Parkinson's disease (PD) is currently the second most common progressive neurodegenerative disorder in the world. Although current science has not been able to discern the exact causes of $\mathrm{PD}$, there is an increasing evidence that genetic factors contribute to the etiology of PD [1].

Certain mutations and risk variants of the gene leucinerich repeat kinase 2 (LRRK2; PARK8) are very common in the PD-afflicted population, accounting for approximately $5-15 \%$ of familial and sporadic PD [2]. Besides, the prevalence differs among different populations $[3,4]$. For instance, the reported proportion of G2019S carriers [5-7] in Ashkenazi Jews and North African Arabs is more than 30\%, whereas it is nearly nonexistent in Asians. Likewise, in Asian populations, the mostly common variants are G2385R and R1628P $[8,9]$. Between these two variants, it is the association between R1628P and PD; however, that is inconclusive. Other studies
[10-13] have found that R1628P is a contributing factor to PD in mainland China, Taiwan, Malaysia, and Thailand. Conversely, a large multicenter study [14] and several other investigators [15-17] did not identify any associated risk of R1628P in either East Asian, Caucasian, or Arab-Berber cohorts.

In order to evaluate the association between R1628P and $\mathrm{PD}$, we used meta-analytic methods to assess the possible role of R1628P in PD in a worldwide context.

\section{Materials and Methods}

2.1. Search Strategy. We searched electronic databases including Embase, PubMed, Cochrane Library, Web of knowledge, Wangfang database, and CNKI. We employed this search method until February 1st, 2017, using a combination of the following keywords, LRRK2, R1628P, PD, and Parkinso*, in both English and Chinese. Reference 
lists and personal communications of authors were also referred as sources to include articles cited elsewhere. All searched publications were imported to endnote for further management. Only one copy of the same papers, which were included in different databases, was kept to avoid duplication.

2.2. Selection Criteria. The following criteria were used to discern the eligibility of a study: (1) the article had to be a casecontrol study, with the exception of reviews, case reports, editorials, or functional research; (2) all PD patients were diagnosed according to UKBB or other accepted criteria with the exception that positive family history was not a part of the exclusion criteria; (3) all controls were free of symptoms suggestive of PD or other neurological disorders; (4) results of $\mathrm{R} 1628 \mathrm{P}$ sequencing were reported both in patients and in controls. We excluded the following studies: (1) duplicate publications and articles which used an overlapping sample and (2) lack of original and sufficient data to calculate the OR.

2.3. Data Extraction and Quality Assessment. To select studies for further assessment, two authors independently scanned the abstracts, titles, or both sections of each retrieved record. All potentially relevant articles were investigated in full. For studies satisfying the aforesaid criteria, two authors independently extracted the following data: year of publication, first author's surname, ethnicity of participants, participant's country of origin, number of R1628P carriers, total number of PD patients and healthy controls, and finally the age and gender ratio for both PD patients and controls. The qualities of the included studies were evaluated by the Newcastle-Ottawa Scale (NOS) [18], of which the genotyping method was an essential part in quality rating process.

2.4. Statistical Analysis. In order to assess the strength of association between LRRK2 R1628P and PD, the outcome was expressed as odds ratio (OR) along with a corresponding 95\% confidence interval (CI). Heterogeneity across individual studies was identified using a standard $Q$ test with a significance level of $\alpha=0.1$ and $I^{2}$. If heterogeneity did not exist $(Q>0.10)$ or the severity of heterogeneity was accepted $\left(I^{2} \leq 50 \%\right)$, then the fixed effect model was adopted to calculate the pooled OR value. Otherwise, the random effect model was used. Funnel-plot analysis was used to assess the reporting bias. All analyses were carried out by using the Review Manager software package v.5.3 (The Cochrane Collaboration, Oxford, England). In addition, subgroup analysis was performed according to ethnicity for all studies. In order to assess the stability of the results, sensitivity analysis was performed by removing each individual study from the combined total and then reanalyzing the remainder.

\section{Results}

3.1. Literature Selection and Study Characteristics. During the initial selection stage, we extracted 148 potentially relevant

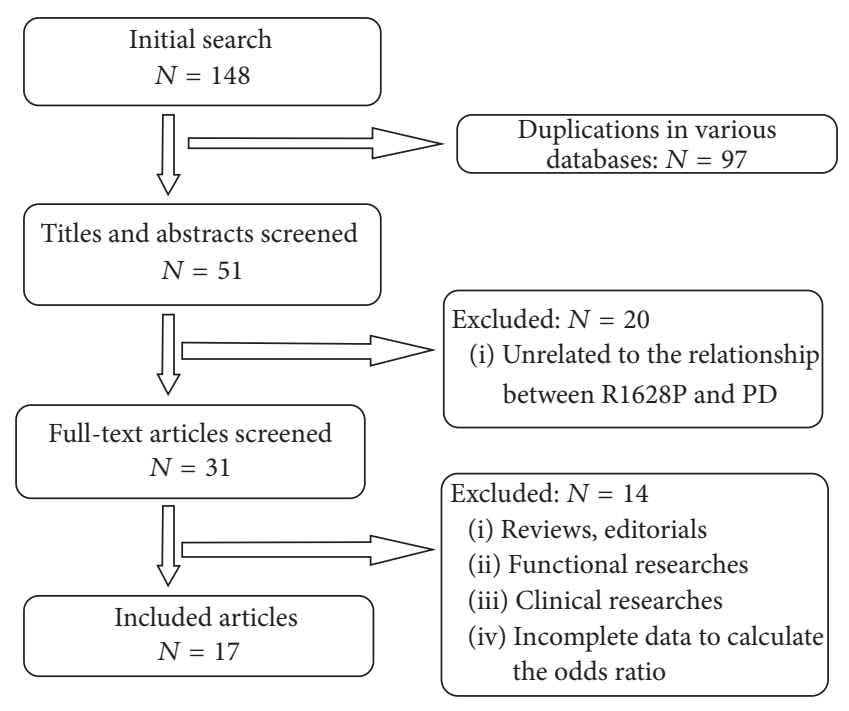

FIGURE 1: Flow chart of included publications.

articles from databases stated above. First, we excluded 97 due to duplication in different databases. Then, 20 publications were excluded for irrelevance to both PD and R1628P, which was determined after screening the titles and abstracts. 14 papers were not included for lack of sequencing results of R1628P both in PD patients and in controls, or the data was not enough to calculate the OR. This process culminated to a total of 17 eligible studies included in qualitative analysis and 16 studies included in the further quantitative analysis. The flow chart illustrating the selection procedure is presented in Figure 1. The main characteristics of the included studies are summarized in Table 1 . The NOS scores of the included publications were rated from 7 to 9 stars, which attested to their high quality.

3.2. Data Synthesis. All of the 17 included studies pertained to Asian populations. The pooled OR for R1628P in Asian was 1.83 (95\% CI: 1.57, 2.13) (Figure 2). The funnel plot was symmetry (Figure 3 ). In a secondary analytic step, we divided all participants into Chinese and non-Chinese groups. Notably, among these chosen databases, the publication by Ross et al. [19] contained both Chinese and non-Chinese populations, which were analyzed individually. Within the Chinese population, there were 6738 cases of PD and 5767 controls. The pooled OR of the Chinese cohort demonstrates a significantly associated risk of R1628P, with the value of 1.84 (95\% CI: 1.56, 2.18) (Figure 2). Among the non-Chinese subgroup, there were 2537 PD subjects and 2347 controls. Since no carriers were screened in Japanese [1924], we conducted the meta-analysis using the other four publications. These publications were studied in Malaysian, Indian, Korean, and Thailand populations. While only the Thailand population demonstrated a significant, independent association, the pooled OR of R1628P in the combined nonChinese group was 1.79 (95\% CI: 1.27, 2.52) (Figure 2). During the sensitivity analysis, there were no significant changes to the outcomes. 
TABLE 1: Attributes of all included studies.

\begin{tabular}{|c|c|c|c|c|c|c|c|}
\hline Publications & Ethnicity & Region & $\begin{array}{c}\text { Total number } \\
\text { of } \\
\text { cases/controls }\end{array}$ & $\begin{array}{l}\text { R1628P carriers of } \\
\text { case/control }\end{array}$ & Age of case/control & $\begin{array}{l}\text { Male ratio of } \\
\text { case/control }\end{array}$ & NOS \\
\hline $2008 \mathrm{Lu}$ et al. [17] & Chinese & Taiwan & $843 / 543$ & $62 / 20$ & $65.7 \pm 11.8 / 51.9 \pm 18.4$ & $58.9 / 41.3$ & 8 \\
\hline 2008 Ross et al. [19] & Chinese & $\begin{array}{l}\text { Taiwan and } \\
\text { Singapore }\end{array}$ & $1079 / 907$ & $66 / 31$ & NA & NA & 8 \\
\hline 2008 Ross et al. [19] & Non-Chinese & Japan & $151 / 95$ & $0 / 0$ & NA & NA & 8 \\
\hline 2008 Tan et al. (2) [20] & Chinese & Singapore & $246 / 243$ & $21 / 8$ & $66 \pm 12 / 62 \pm 10$ & $56.0 / 54.0$ & 8 \\
\hline 2008 Tan et al. (1) [21] & Non-Chinese & $\begin{array}{c}\text { Malaysia } \\
\text { and Indian }\end{array}$ & $192 / 265$ & $3 / 6$ & $60.3 \pm 6.4 / 64.5 \pm 3.7$ & NA & 9 \\
\hline 2009 Zhang et al. [22] & Chinese & China & $600 / 459$ & $43 / 11$ & $\mathrm{NA} / 53.4 \pm 13.4$ & $57.0 / 56.0$ & 7 \\
\hline 2009 Yu et al. [23] & Chinese & China & $328 / 300$ & $17 / 6$ & $\mathrm{NA} / 58.2 \pm 10.3$ & $54.3 / 57.7$ & 9 \\
\hline 2009 Zabetian et al. [24] & Non-Chinese & Japan & $631 / 320$ & $0 / 0$ & NA & NA & 9 \\
\hline 2010 Kim et al. [15] & Non-Chinese & Korea & $383 / 379$ & $3 / 1$ & NA & NA & 8 \\
\hline 2011 Pulkes et al. [25] & Thai/Chinese & Thai & $154 / 156$ & $15 / 5$ & NA & $56.5 / \mathrm{NA}$ & 8 \\
\hline 2012 Zhou et al. [16] & Chinese & China & $202 / 212$ & $2 / 5$ & $\begin{array}{c}62.68 \pm 10.69 / 62.87 \pm \\
10.44\end{array}$ & $52.5 / 59.0$ & 8 \\
\hline $2013 \mathrm{Fu}$ et al. [13] & Chinese & China & $446 / 403$ & $47 / 21$ & $60.82 \pm 11.20 / 59.21 \pm 9.3$ & $59.0 / 62.0$ & 9 \\
\hline $2013 \mathrm{Wu}$-Chou et al. [12] & Chinese & Taiwan & $747 / 461$ & $58 / 18$ & NA & NA & 8 \\
\hline 2013 Cai et al. [26] & Chinese & China & $510 / 550$ & $29 / 19$ & $58.4 \pm 11.0 / 59.34 \pm 11.3$ & $59.4 / 58.2$ & 9 \\
\hline 2013 Wu et al. [27] & Chinese & Taiwan & $573 / 503$ & $34 / 22$ & $62.1 \pm 11.5 / 59.4 \pm 12.9$ & $55.3 / 50.7$ & 8 \\
\hline 2014 Pulkes et al. [11] & Non-Chinese & Thai & $485 / 480$ & $54 / 29$ & $65.0 \pm 12.0 / 71.0 \pm 7.0$ & $46.0 / 61.0$ & 9 \\
\hline 2014 Gopalai et al. [10] & Non-Chinese & Malaysia & $695 / 507$ & $43 / 18$ & $57.4 \pm 11.8 / 59.3 \pm 9.4$ & $60.0 / 51.0$ & 8 \\
\hline 2015 Guo et al. [28] & Chinese & China & $1019 / 1030$ & $49 / 44$ & $57.9 \pm 12.3 / 57.2 \pm 16.4$ & $58.5 / 48.0$ & 9 \\
\hline
\end{tabular}

NOS: Newcastle-Ottawa Scale. NA: not available.

\section{Discussion}

In the meta-analysis, a combination of $9275 \mathrm{PD}$ patients and 8114 controls was studied. All included publications were of high quality according to NOS and the results were stable during sensitive analysis. Furthermore, there was no publication reporting bias, as shown by the symmetric funnel plot. It is believed that the final results are credible. The included studies were conducted in mainland China, Taiwan, Singapore, Japan, Malaysia, India, Korea, and Thailand. We found that R1628P carriers were reported as simply being a part of the general Asian population and then subsequently divided them into Chinese and non-Chinese for further stratified analysis. Even though a variety of Asian countries were included, the heterogeneity of the sample was accepted with $I^{2}$ less than 50\%. Therefore, based on the evidence, we found that $L R R K 2 \mathrm{R} 1628 \mathrm{P}$ was a risk factor for PD in Asian, 'both Chinese and non-Chinese, populations.

Although the cause of PD is unclear, it is accepted that its etiology is the interaction of genetic and environmental factors, along with genetic insights which provide a way to explore the molecular causes of PD. As a result, more and more attention has been focused on the relationship between genetics and PD. LRRK2, one of the genes related to autosomal dominant form of PD, belongs to a member of the ROCO protein family [29] and has five major functional domains [30]: a leucine-rich repeat (LRR); a Roc domain
(Ras in complex proteins); a COR domain (C-terminal of Roc); a TyrKc domain (tyrosine kinase catalytic), and WD40 domain. The variations of LRRK2 account for approximately $5-15 \%$ of familial and sporadic PD [2]. Additionally, further studies show that various variants, such as G2019S and G2385R, have genetic correlations and heterogeneity. This means that different variations, including mutations and polymorphism, may play a diverse role depending on the given population.

R1628P, a substitution of a polar arginine $(R)$ with a neutral nonproline $(\mathrm{P})$, is located in the COR domain of LRRK2. This kind of substitution could change the secondary structure of LRRK2 and influence the interaction between the other domains of LRRK2 or different external proteins, which ultimately influences the kinase activity [31, 32]. In fact, a study in our team once reported that R1628P was not associated with PD risk in mainland China [28] $(\mathrm{PD}=1019$, $N=1030$ ), which was consistent with $[11,27]$ or contrary to $[12,13,22]$ other similar studies conducted. We suppose that a larger number of participants would be helpful for observing the actual results. To draw a comprehensive understanding, we performed a meta-analysis to evaluate the contribution of LRRK2 R1628P to PD worldwide. And we found LRRK2 R1628P was a risk factor in Asian ethnicities, which may help for further pathogenetic exploring of $L R K K 2$, especially in the Asian population. Additionally, Shu's study identified that R1628P could be an example that explains the geneticenvironmental interaction. Instead of upregulation of the 


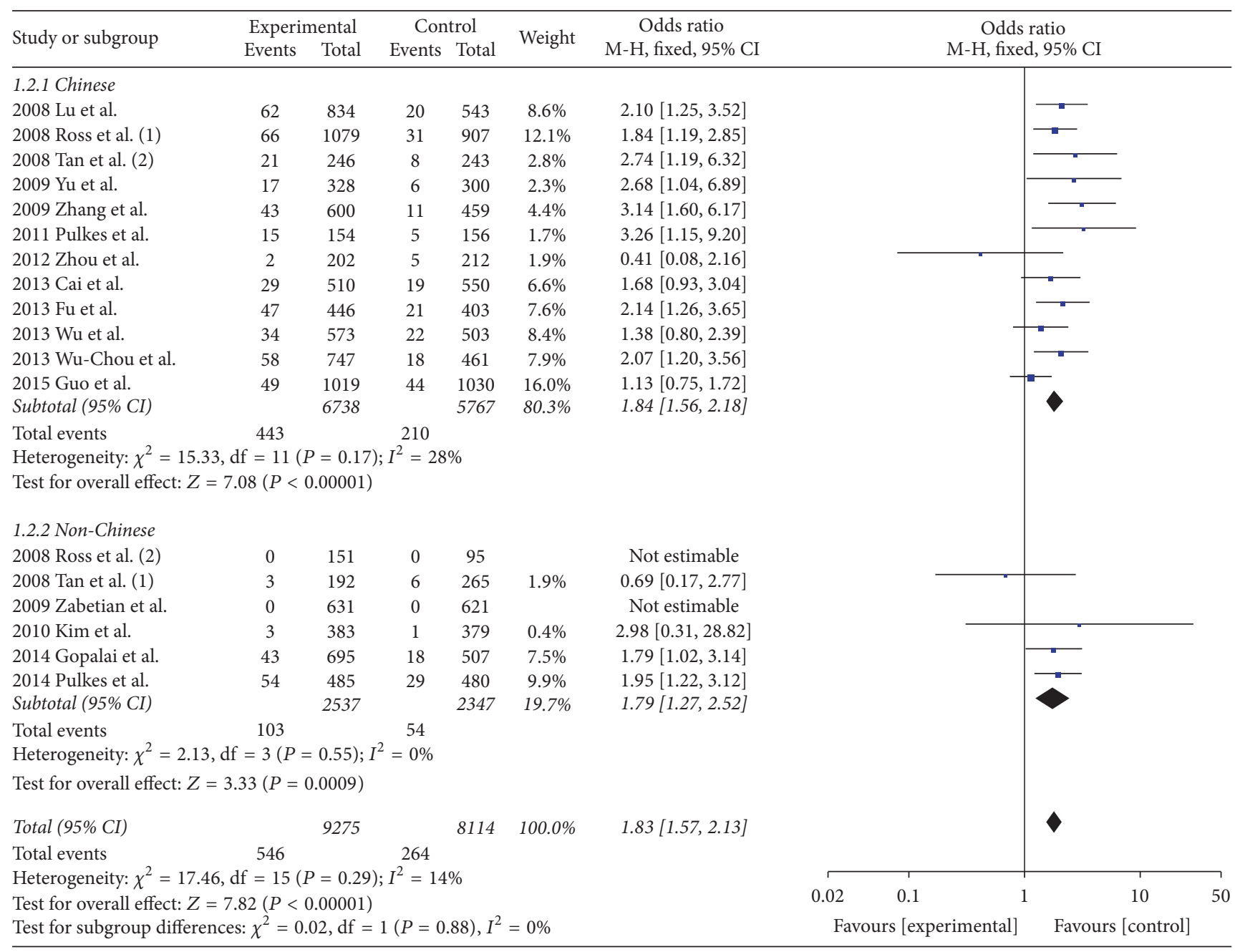

FIgURE 2: Forest plot of R1628P in PD.

kinase activity of LRRK2 directly, it was found that the status of R1628P in LRRK2 provides a potential "two-hit" target: firstly environmentally toxic induced Cdk5 activation and then $\mathrm{Cdk} 5$ phosphorylating the adjacent amino residue S1627 of the R1628P mutation; thus LRRK2 kinase was activated which leads to the death of neurons.

The International Parkinson and Movement Disorder Society (MDS) Task Force proposed that PD should be divided into three stages [33]: preclinical PD, prodromal PD, and clinical PD. The stage of prodromal PD [34] refers to the presentation of symptoms or signs of PD neurodegeneration with absence of classic motor parkinsonism. Genetics findings, combined with age, environmental risk factors, and other diagnostic marker tests, play a vital role in the diagnosis of prodromal PD $[34,35]$. We found $L R R K 2 \mathrm{R} 1628 \mathrm{P}$ was a risk factor in Asian populations, both Chinese and non-Chinese. This may help for further exploring etiology and biomarker of $\mathrm{PD}$, especially in Asian population.

A few limitations of this study should be acknowledged: firstly, as all data were extracted from previously published papers, we do not know the effects of the unpublished articles.
Secondly, some articles [14, 36, 37], which otherwise met the eligibility criteria, were excluded for insufficient data. Thirdly, other confounding factors, like age, gender, and age at onset, may be affecting the outcomes and prevalence of PD.

In conclusion, our research showed a significant association between R1628P and the susceptibility to PD in the Asian population, both Chinese and non-Chinese. Further investigation of high quality, large cohort studies and function researches is needed to elaborate this relationship.

\section{Conflicts of Interest}

No conflicts of interest exist in the submission and publication of this manuscript.

\section{Acknowledgments}

This work was supported by grants from the National Natural Science Foundation of China (no. 81401059, no. 31500999, 


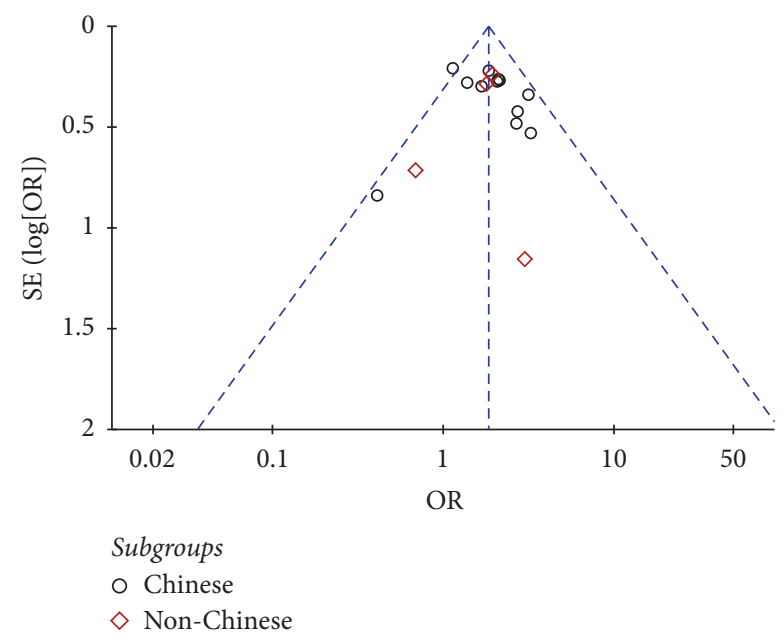

FIGURE 3: Funnel plot of R1628P in PD.

and no. 81430023), the National Key Plan for Scientific Research and Development of China (no. 2016YFC1306000), and Hunan Provincial Innovation Foundation For Postgraduate (no. CX2017B066).

\section{References}

[1] L. V. Kalia and A. E. Lang, "Parkinson's disease," The Lancet, vol. 386, no. 9996, pp. 896-912, 2015.

[2] J. C. Dächsel and M. J. Farrer, "LRRK2 and Parkinson disease," Archives of Neurology, vol. 67, no. 5, pp. 542-547, 2010.

[3] A. Di Fonzo, C. Tassorelli, M. De Mari et al., "Comprehensive analysis of the LRRK2 gene in sixty families with Parkinson's disease," European Journal of Human Genetics, vol. 14, no. 3, pp. 322-331, 2006.

[4] C. Paisán-Ruíz, P. Nath, N. Washecka, J. R. Gibbs, and A. B. Singleton, "Comprehensive analysis of LRRK2 in publicly available Parkinson's disease cases and neurologically normal controls," Human Mutation, vol. 29, no. 4, pp. 485-490, 2008.

[5] S. Lesage, A. Dürr, M. Tazir et al., "LRRK2 G2019S as a cause of Parkinson's disease in North African Arabs," The New England Journal of Medicine, vol. 354, no. 4, pp. 422-423, 2006.

[6] M. M. Hulihan, L. Ishihara-Paul, J. Kachergus et al., "LRRK2 Gly2019Ser penetrance in Arab-Berber patients from Tunisia: a case-control genetic study," The Lancet Neurology, vol. 7, no. 7, pp. 591-594, 2008.

[7] T. Peeraully and E. K. Tan, "Genetic variants in sporadic parkinson's disease: East vs west," Parkinsonism \& Related Disorders, vol. 18, supplement 1, pp. S63-S65, 2012.

[8] M. J. Farrer, J. T. Stone, C.-H. Lin et al., "Lrrk2 G2385R is an ancestral risk factor for Parkinson's disease in Asia," Parkinsonism \& Related Disorders, vol. 13, no. 2, pp. 89-92, 2007.

[9] A. Di Fonzo, Y. H. Wu-Chou, C. S. Lu et al., "A common missense variant in the LRRK2 gene, Gly2385Arg, associated with Parkinson's disease risk in Taiwan," neurogenetics, vol. 7, no. 3, pp. 133-138, 2006.

[10] A. A. Gopalai, S.-Y. Lim, J. Y. Chua et al., "LRRK2 G2385R and R1628P Mutations Are Associated with an Increased Risk of Parkinson's Disease in the Malaysian Population," BioMed Research International, vol. 2014, Article ID 867321, 2014.
[11] T. Pulkes, C. Papsing, A. Thakkinstian et al., "Confirmation of the association between LRRK2 R1628P variant and susceptibility to Parkinson's disease in the Thai population," Parkinsonism \& Related Disorders, vol. 20, no. 9, pp. 1018-1021, 2014.

[12] Y.-H. Wu-Chou, Y.-T. Chen, T.-H. Yeh et al., "Genetic variants of SNCA and LRRK2 genes are associated with sporadic PD susceptibility: a replication study in a Taiwanese cohort," Parkinsonism \& Related Disorders, vol. 19, no. 2, pp. 251-255, 2013.

[13] X. Fu, Y. Zheng, H. Hong et al., "LRRK2 G2385R and LRRK2 R1628P increase risk of Parkinson's disease in a Han Chinese population from Southern Mainland China," Parkinsonism \& Related Disorders, vol. 19, no. 3, pp. 397-398, 2013.

[14] O. A. Ross, A. I. Soto-Ortolaza, M. G. Heckman et al., "Association of LRRK2 exonic variants with susceptibility to Parkinson's disease: a case-control study," The Lancet Neurology, vol. 10, no. 10, pp. 898-908, 2011.

[15] J. M. Kim, J. Y. Lee, H. J. Kim et al., “The LRRK2 G2385R variant is a risk factor for sporadic Parkinson's disease in the Korean population," Parkinsonism \& Related Disorders, vol. 16, no. 2, pp. 85-88, 2010.

[16] Y. Zhou, X. Luo, F. Li et al., "Association of Parkinson's disease with six single nucleotide polymorphisms located in four PARK genes in the northern Han Chinese population," Journal of Clinical Neuroscience, vol. 19, no. 7, pp. 1011-1015, 2012.

[17] C.-S. Lu, Y.-H. Wu-Chou, M. van Doeselaar et al., "The LRRK2 Arg1628Pro variant is a risk factor for Parkinson's disease in the Chinese population," Neurogenetics, vol. 9, no. 4, pp. 271-276, 2008.

[18] A. Stang, "Critical evaluation of the Newcastle-Ottawa scale for the assessment of the quality of nonrandomized studies in metaanalyses," European Journal of Epidemiology, vol. 25, no. 9, pp. 603-605, 2010.

[19] O. A. Ross, Y.-R. Wu, M.-C. Lee et al. et al., "Analysis of Lrrk2 R1628P as a risk factor for Parkinson's disease," Annals of Neurology, vol. 64, no. 1, pp. 88-92, 2008.

[20] E. K. Tan, M. Tang, L. C. Tan et al., "Lrrk2 R1628P in nonChinese Asian races," Annals of Neurology, vol. 64, no. 4, pp. 472-473, 2008.

[21] E. K. Tan, L. C. Tan, H. Q. Lim et al., "LRRK2 R1628P increases risk of Parkinson's disease: replication evidence," Human Genetics, vol. 124, no. 3, pp. 287-288, 2008.

[22] Z. Zhang, J.-M. Burgunder, X. An et al., "LRRK2 R1628P variant is a risk factor of Parkinson's disease among Han-Chinese from mainland China," Movement Disorders, vol. 24, no. 13, pp. 19021905, 2009.

[23] L. Yu, F. Hu, X. Zou et al., "LRRK2 R1628P contributes to Parkinson's disease susceptibility in Chinese Han populations from mainland China," Brain Research, vol. 1296, pp. 113-116, 2009.

[24] C. P. Zabetian, M. Yamamoto, A. N. Lopez et al., "LRRK2 mutations and risk variants in Japanese patients with Parkinson's disease," Movement Disorders, vol. 24, no. 7, pp. 1034-1041, 2009.

[25] T. Pulkes, C. Papsing, S. Mahasirimongkol, M. Busabaratana, K. Kulkantrakorn, and S. Tiamkao, "Frequencies of LRRK2 variants in Thai patients with Parkinson's disease: evidence for an R1628P founder," Journal of Neurology, Neurosurgery \& Psychiatry, vol. 82, no. 10, pp. 1179-1180, 2011.

[26] J. Cai, Y. Lin, W. Chen et al., "Association between G2385R and R1628P polymorphism of LRRK2 gene and sporadic Parkinson's disease in a Han-Chinese population in south-eastern China," Neurological Sciences, vol. 34, no. 11, pp. 2001-2006, 2013. 
[27] Y.-R. Wu, K.-H. Chang, W.-T. Chang et al., "Genetic variants of LRRK2 in Taiwanese Parkinson's disease," PLoS ONE, vol. 8, no. 12, Article ID e82001, 2013.

[28] J.-F. Guo, K. Li, R.-L. Yu et al., "Polygenic determinants of Parkinson's disease in a Chinese population," Neurobiology of Aging, vol. 36, no. 4, pp. 1765.el-1765.e6, 2015.

[29] L. Bosgraaf and P. J. M. van Haastert, "Roc, a Ras/GTPase domain in complex proteins," Biochimica et Biophysica Acta, vol. 1643, no. 1-3, pp. 5-10, 2003.

[30] A. Zimprich, S. Biskup, P. Leitner et al., "Mutations in LRRK2 cause autosomal-dominant parkinsonism with pleomorphic pathology," Neuron, vol. 44, no. 4, pp. 601-607, 2004.

[31] S. Saiki, S. Sato, and N. Hattori, "Molecular pathogenesis of Parkinson's disease: update," Journal of Neurology, Neurosurgery \& Psychiatry, vol. 83, no. 4, pp. 430-436, 2012.

[32] S. L. Chan, D. C. Angeles, and E.-K. Tan, "Targeting leucinerich repeat kinase 2 in Parkinson's disease," Expert Opinion on Therapeutic Targets, vol. 17, no. 12, pp. 1471-1482, 2013.

[33] D. Berg, R. B. Postuma, B. Bloem et al., “Time to redefine PD? Introductory statement of the MDS Task Force on the definition of Parkinson's disease," Movement Disorders, vol. 29, no. 4, pp. 454-462, 2014.

[34] D. Berg, R. B. Postuma, C. H. Adler et al., "MDS research criteria for prodromal Parkinson's disease," Movement Disorders, vol. 30, no. 12, pp. 1600-1611, 2015.

[35] M. A. Nalls, C. Y. McLean, J. Rick et al., "Diagnosis of Parkinson's disease on the basis of clinical and genetic classification: A population-based modelling study," The Lancet Neurology, vol. 14, no. 10, article no. 123, pp. 1002-1009, 2015.

[36] E. K. Tan, R. Peng, Y. Y. Teo et al., "Multiple LRRK2 variants modulate risk of Parkinson disease: a Chinese multicenter study," Human Mutation, vol. 31, no. 5, pp. 561-568, 2010.

[37] C. Wang, Y. Cai, Z. Zheng et al., "Penetrance of LRRK2 G2385R and R1628P is modified by common PD-associated genetic variants," Parkinsonism \& Related Disorders, vol. 18, no. 8, pp. 958-963, 2012. 


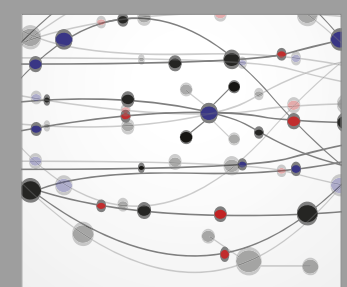

The Scientific World Journal
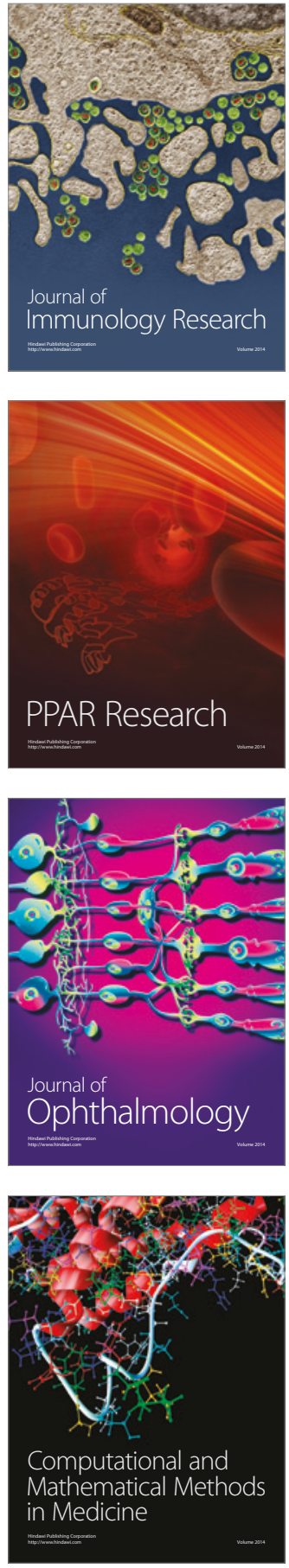

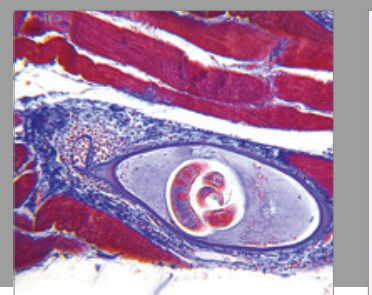

Gastroenterology Research and Practice
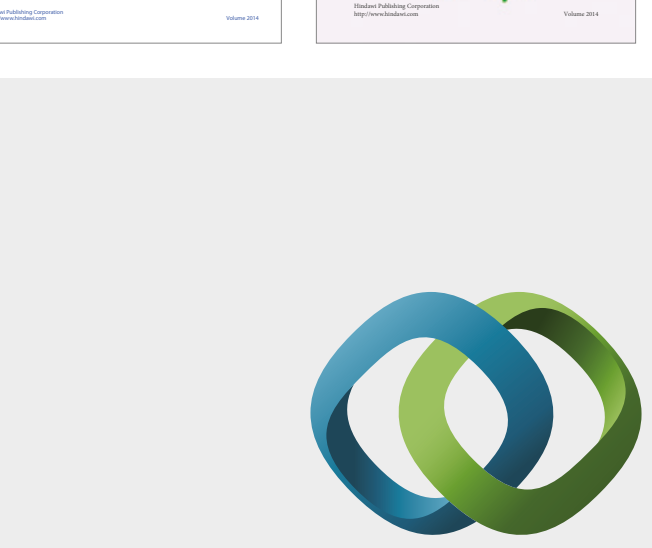

\section{Hindawi}

Submit your manuscripts at

https://www.hindawi.com
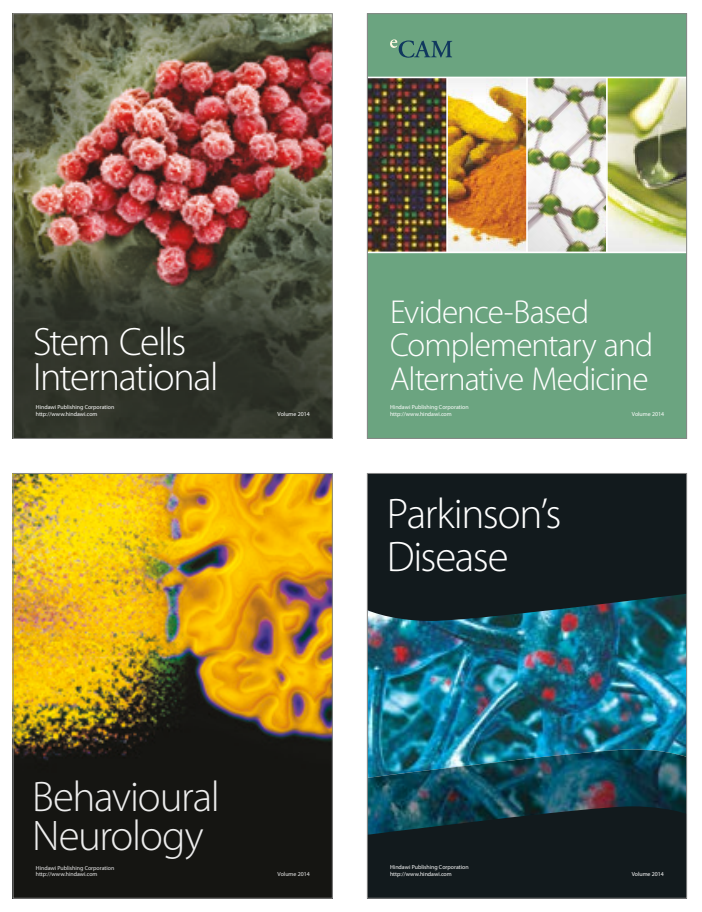
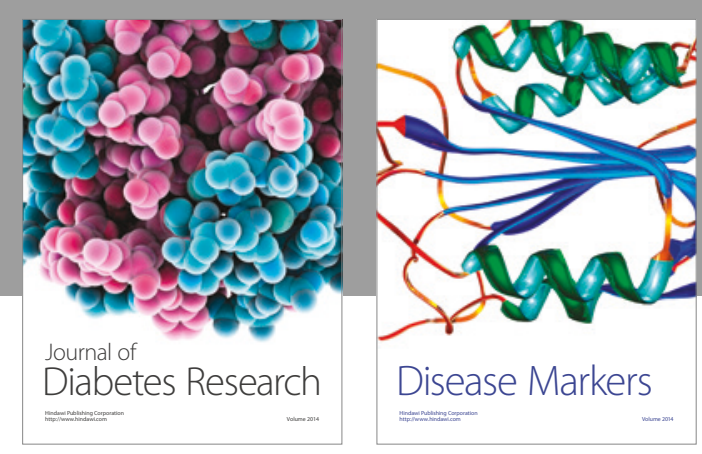

Disease Markers
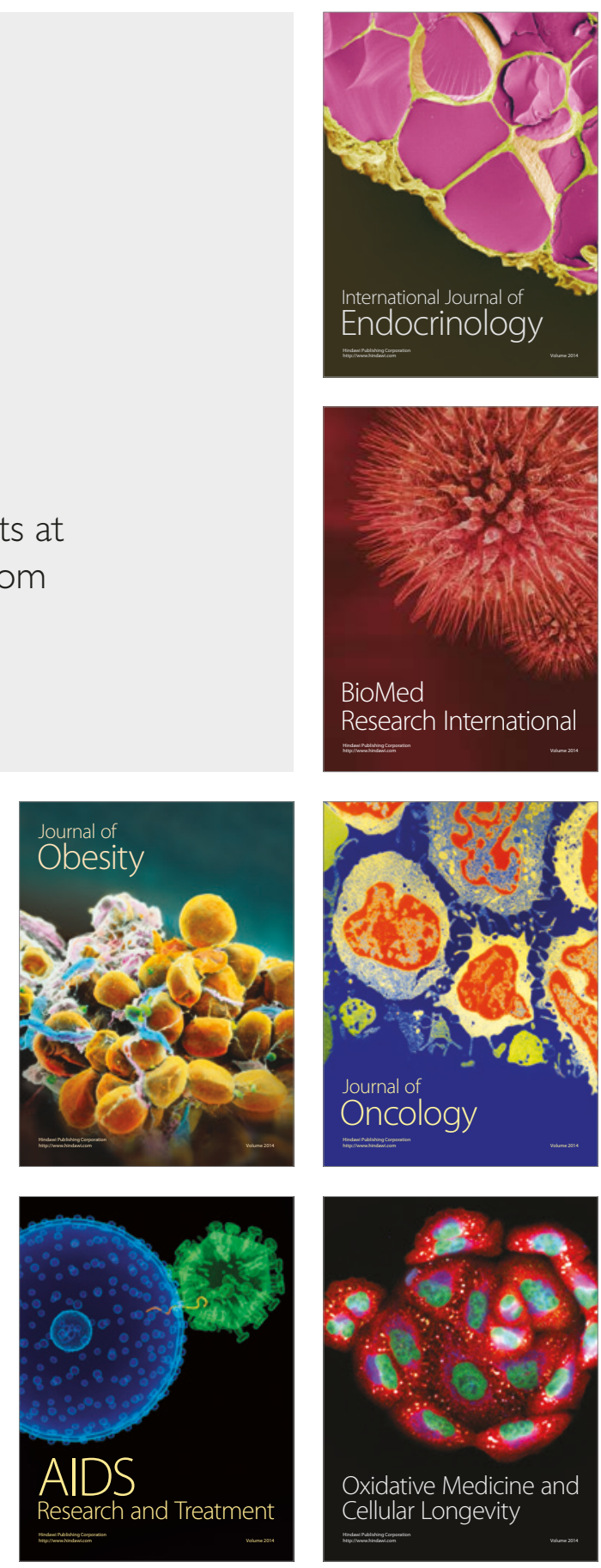